\title{
CONFIRMACIÓN DEL MÉTODO DE AYUDA DIAGNÓSTICA DE LA DINÁMICA CARDIACA DE APLICACIÓN CLÍNICA DESARROLLADO CON BASE EN LA TEORÍA DE LA PROBABILIDAD
}

\author{
Javier Rodríguez ${ }^{1}$, Catalina Correa ${ }^{2}$, Signed Prieto ${ }^{3}$, Pedro Bernal ${ }^{4}$, Germán Forero ${ }^{5}$, Gabriel Salazar ${ }^{6}$, \\ SARITH VITERY ${ }^{7}$, LUISA ALVAREZ ${ }^{8}$, GERMÁN PUERTA ${ }^{9}$ \\ ${ }^{1} \mathrm{MD}$, Director del Grupo Insight- Centro de Investigaciones Clinica del Country. Director de la Linea de Profundización \\ e Internado Especial: Física y Matemáticas Aplicadas a la Medicina, Universidad Militar Nueva Granada. \\ ${ }^{2}$ PSY, Investigadora Grupo Insight-Centro de Investigaciones Clínica del Country. Profesora de la Línea de Profundización \\ e Internado Especial: Física y Matemáticas Aplicadas a la Medicina, Universidad Militar Nueva Granada. \\ ${ }^{3}$ Investigadora Grupo Insight- Centro de Investigaciones Clinica del Country. ${ }^{4}$ Investigador Grupo Insight- Centro \\ de Investigaciones Clínica del Country. ${ }^{5}$ MD, Neurocirujano. Docente Universidad Militar Nueva Granada. \\ ${ }^{6}$ Cardiólogo, Fundación Cardio Infantil - Instituto de Cardiología. ${ }^{7}$ Internado Especial: Física y Matemáticas Aplicadas \\ a la Medicina, Universidad Militar Nueva Granada-Centro de Investigaciones Clínica del Country. ${ }^{8}$ PSY, Investigadora \\ Grupo Insight-Centro de Investigaciones Clínica del Country. ${ }^{9}$ Internado Especial: Física y Matemáticas Aplicadas a \\ la Medicina, Universidad Militar Nueva Granada- Centro de Investigaciones Clínica del Country.
}

\begin{abstract}
Resumen
A partir de la teoría de probabilidad y los sistemas dinámicos se desarrolló previamente una nueva metodología de ayuda diagnóstica para el Holter con base en la teoría de la probabilidad. El propósito de este trabajo es evaluar su concordancia diagnóstica en casos normales y con enfermedad aguda. Se tomaron 15 Holters normales y 100 con diferentes patologías cardiacas de pacientes mayores a 20 años. Se establecieron rangos de frecuencias cardiacas y de número de latidos por hora y se calculó la probabilidad de estos rangos. Los valores obtenidos se analizaron de acuerdo con los parámetros diagnósticos establecidos en la metodología previamente desarrollada para diferenciar normalidad de enfermedad. Finalmente se evaluó sensibilidad, especificidad y coeficiente Kappa de la evaluación física matemática respecto al diagnóstico convencional para los casos normales y con enfermedad aguda.

Los Holter con enfermedad aguda presentaron entre 6 y 13 rangos de frecuencias y los normales entre 13 y 21 rangos. La probabilidad máxima de latidos por hora en normalidad presentó cuatro casos con probabilidad menor o igual a 0,217 o mayor o igual a 0,304, mientras que para enfermedad aguda todos tuvieron valores mayores o iguales a 0,304. De los Holter, 5 con enfermedad aguda, y 2 normales presentaron un número de latidos menor a 3.000. La suma de las probabilidades de las dos frecuencias más probables se encontró entre 0,203 y 0,379 para los normales y entre 0,333 y 0,652 para los Holters con patologías agudas. Los valores de sensibilidad y especificidad fueron de 100\% Y 73,3\% y el coeficiente Kappa de 0,86.

Se confirmó que la metodología desarrollada con base en la teoría de la probabilidad revela una autoorganización del sistema cardiaco que permite diferenciar normalidad de enfermedad aguda y evidenciar la evolución entre ambos a nivel clínico.
\end{abstract}

Palabras clave: Holter, probabilidad, ayuda diagnóstica.

* Correspondencia: Javier Rodríguez grupoinsight2025@yahoo.es. Dirección postal: Cra. 79 B \# 51-16 Sur, Interior 5 apto 102. Teléfono: 45275 41, Bogotá, Colombia.

Recibido: agosto 10 de 2011 Aceptado: noviembre 30 de 2011 


\title{
CONFIRMATION OF THE METHODOLOGY OF DIAGNOSTIC AID FOR THE CARDIAC DYNAMICS FOR CLINICAL APPLICATION DEVELOPED BASED ON THE PROBABILITY THEORY
}

\begin{abstract}
Summary
Starting from the probability theory and dynamic systems, a novel methodology of diagnostic aid for the Holter test was developed previously, based on the probability theory. The aim of this paper is to assess its diagnostic concordance in normal and acutely ill cases.

Fifteen normal Holter tests and 100 with different cardiac pathologies from patients older than 20 years were taken. Cardiac rates ranges and number of beats per hour were determined and the probability of theses ranges was estimated. The obtained values were analyzed according to the diagnostic parameters established in the previously developed methodology in order to differentiate the normal status from the disease. Finally, sensitivity, specificity and Kappa coefficient of the physical mathematical evaluation with regard to the conventional diagnosis for normal and acutely ill cases were assessed.

The Holter tests with acute disease showed between 6 and 13 ranges of frequencies, and the normal ones between 13 and 21 ranges. The maximum probability of beats per hour in normality presented four cases with probability less then or equal to 0.217 or higher than or equal to 0.304 , while for acute disease all had values higher than or equal to 0.304. From among the Holter tests, 5 with acute disease and 2 normal presented a number of beats lower than 3.000. The sum of probabilities of the most probable frequencies was between 0.203 and 0.379 for the normal ones and between 0.333 and 0.652 for the Holter tests with acute pathologies. Sensitivity and specificity values were $100 \%$ and $73.3 \%$, and the Kappa coefficient 0.86 .

It was confirmed that the methodology developed based on the probability theory reveals an autoorganization of the cardiac system which allows to differentiate between normality and acute disease and to evidence the evolution between both at the clinical level.
\end{abstract}

Key words: Holter test, probability, diagnostic aid.

\section{CONFIRMAÇÃO DO MÉTODO DE AJUDA DIAGNÓSTICA DA DINÂMICA CARDÍACA DE APLICAÇÃO CLÍNICA DESENVOLVIDO COM BASE NA TEORIA DA PROBABILIDADE}

\begin{abstract}
Resumo
A partir da teoría de probabilidade e dos sistemas dinâmicos desenvolveu-se previamente uma nova metodologia de ajuda diagnóstica para o Holter com base na teoría da probabilidade. O propósito deste trabalho é avaliar sua concordância diagnóstica em casos normais e com doença aguda. Foram tomados Holters normais e 100 com diferentes patologias cardíacas de pacientes maiores de 20 anos. Estabeleceram-se intervalos de frequências cardíacas e de número de batidas por hora e calculou-se a probabilidade destes intervalos. Os valores obtidos foram analisados de acordo com os parâmetros diagnósticos estabelecidos na metodologia previamente desenvolvida para diferenciar normalidade de doença. Finalmente avaliou-se sensibilidade, especificidade e coeficiente Kappa da avaliação física matemática com respeito ao diagnóstico convencional para os casos normais e com doença aguda.

Os Holters com doença aguda apresentaram entre 6 e 13 intervalos de frequências e os normais entre 13 e 21 intervalos. A probabilidade máxima de batidas por hora em normalidade apresentou quatro casos com probabilidade menor ou igual a 0,217 ou maior ou igual a 0,304 , enquanto que
\end{abstract}




\begin{abstract}
para doença aguda todos tiveram valores maiores ou iguais que 0,304 . Dos Holter, 5 com doença aguda, e 2 normais apresentaram um número de batidas menor que 3.000. A soma das probabilidades das duas frequências mais prováveis foi encontrada entre 0,203 e 0,379 para os normais e entre 0,333 e 0,652 para os Holters com patologias agudas. Os valores de sensibilidade e especificidade foram de $100 \%$ e $73,3 \%$ e o coeficiente Kappa de 0,86.

Confirmou-se que a metodologia desenvolvida com base na teoria da probabilidade revela una autoorganização do sistema cardíaco que permite diferenciar normalidade de doença aguda e evidenciar a evolução entre ambos a nível clínico.
\end{abstract}

Palavras chave: Holter, probabilidade, ajuda diagnóstica.

\section{Introducción}

La teoría de sistemas dinámicos permite caracterizar el estado y la evolución de los sistemas en el tiempo, al representar geométricamente sus variables en espacios de fases, obteniendo una figura denominada atractor mediante la cual es posible establecer características como la impredecibilidad o predecibilidad del sistema, así como su dimensión fractal $(1,2)$. La teoría de sistemas dinámicos ha sido base para el desarrollo de estudios en cardiología, como el trabajo de Huikuri y cols., quienes lograron superar los predictores de mortalidad convencionales en pacientes con infarto agudo de miocardio con una fracción de eyección menor al 35\% (3). Goldberger et al. (4) establecieron una nueva concepción salud/enfermedad que evidencia que comportamientos excesivamente periódicos o aleatorios se asocian a estados patológicos en tanto que la normalidad se caracteriza por un comportamiento intermedio entre ambos, contradiciendo la concepción homeostática tradicional.

A partir de la concepción de salud y enfermedad de la teoría de sistemas dinámicos, se han desarrollado nuevas metodologías de evaluación en cardiología; por ejemplo, una reinterpretación de esta concepción y la aplicación de la ley de Zipf-Mandelbrot permitieron el desarrollo de una nueva metodología diagnóstica para la monitoria cardiaca fetal $(5,6)$. Así mismo, se han efectuado diferentes trabajos en la evaluación del Holter (7-9); entre ellos, se desarrolló una nueva metodología de evaluación del holter con base en la teoría de la probabilidad, que puede ser empleada como herramienta de ayuda diagnóstica de aplicación clínica (9).

La probabilidad es una medida matemática con la que se cuantifica la posible ocurrencia de un evento en el futuro (10-13). Las leyes de la probabilidad fue- ron aplicadas en el trabajo desarrollado a rangos de aparición de la frecuencia cardiaca y del número de latidos, encontrando que la aplicación consecutiva de 3 parámetros definidos permite la diferenciación entre normalidad y enfermedad. Con esta metodología se caracterizó de una manera física y matemática la dinámica cardiaca, en una forma objetiva y reproducible, revelando una auto-organización matemática que diferencia normalidad de enfermedad y evolución entre las dos; esta metodología es aplicable a individuos mayores de 20 años, proporcionando un diagnóstico preciso para cualquier caso particular, independientemente de la edad, patología e intervenciones (9).

El propósito de este trabajo es realizar una aplicación de la metodología diagnóstica desarrollada con base en la teoría de la probabilidad (9), así como evaluar la concordancia diagnóstica de dicho método respecto al Gold Standard en casos normales y con diferentes patologías agudas, con el fin de confirmar su aplicabilidad clínica a estos casos.

\section{Metodología}

\section{Definiciones}

Rango de frecuencia cardiaca: intervalo de 5 latidos/minuto en el cual se halla el valor de la frecuencia cardiaca.

Rango del número de latidos: rango de 250 latidos en el que se halla el número total de latidos de cada hora.

Probabilidad del Rango: frecuencia de un rango medido $\mathrm{N}_{\mathrm{R}}$, dividido entre el total de repeticiones de los rangos medidos $\mathrm{N}$ :

$$
P(A)=\frac{\text { Repeticiones del rango } r}{\text { Total de repeticiones de los rangos medidos }}=\frac{N_{R}}{N}
$$




\section{Población}

Se analizaron 115 Holters ambulatorios de individuos mayores a 20 años, provenientes de la Fundación Cardio Infantil - Instituto de Cardiología, correspondientes a 15 pacientes con indicaciones de sintomatologías previas como palpitaciones, síncope, taquicardia, fatiga o vértigo, cuyos resultados reportados en el Holter de acuerdo con la evaluación convencional se encontraron dentro de los límites normales y 100 con indicaciones tales como taquicardia, arritmia, fibrilación auricular, disfunción nodal o extrasistolia, que presentaron un diagnóstico patológico en el Holter. Los diagnósticos fueron establecidos por un cardiólogo experto desde los parámetros clínicos convencionales.

\section{Procedimiento}

Se tomaron los valores máximos, mínimos e intermedios de las frecuencias cardiacas cada hora durante el monitoreo electrocardiográfico durante mínimo 21 horas, se dividieron en rangos de cinco latidos por minuto, para calcular la probabilidad del número de frecuencias que se obtienen en cada rango respecto a la totalidad de frecuencias obtenidas en cada Holter, para finalmente contar el número de rangos en cada paciente, la diferencia entre los rangos de los dos valores de frecuencia con mayor probabilidad y la suma de las probabilidades de dichos valores. Adicionalmente se evaluó la probabilidad del número total de latidos por hora a partir de rangos de a 250 latidos, durante mínimo 21 horas, determinando la máxima probabilidad encontrada en cada Holter y se evaluó el número de latidos mínimo y máximo. Los valores obtenidos fueron analizados de acuerdo con los 3 parámetros diagnósticos ya establecidos (9), y que se describen a continuación, para obtener el diagnóstico matemático para cada dinámica:

1. Valores superiores a 17 en el número de rangos de la frecuencia cardiaca son indicadores de normalidad, mientras que valores inferiores a 14 normalmente son característicos de enfermedad. Para determinar el diagnóstico de los valores intermedios se aplica el parámetro 2 , constituido por dos subparámetros que se aplican simultáneamente: a) si se presenta una diferencia mayor o igual a 15 entre los rangos de los dos valores de frecuencia que presentaron mayores valores de probabilidad, se diagnostica enfermedad. b) Un valor igual o menor a 0,217 o mayor o igual a 0,304 presente en la máxima probabilidad del número de latidos es señal de enfermedad. A partir de lo cual se establece la evaluación, de manera que $\mathrm{Si}$ solamente se presenta a, hay enfermedad; Si se presenta a y b, hay enfermedad; Si solamente se presenta b, hay evolución a la enfermedad; $\mathrm{Si}$ se presenta $b$ y un número de latidos menor a 3,000 o mayor a 6.250, hay enfermedad. 3. cuando se presentan valores mayores a 0,319 en la suma de las dos probabilidades más frecuentes se diagnostica enfermedad, cuando se presenta una de las siguientes condiciones: los subparámetros a y b del parámetro 2 también presentaron valores asociados a enfermedad; el parámetro b es característico de enfermedad; o bien se registra un número mayor a 6.250 latidos o menor a 3.000 en una hora, y adicionalmente el parámetro $\mathrm{b}$ se asocia a enfermedad (9).

\section{Análisis estadístico}

Se tomó como Gold-Estándar el diagnóstico de normalidad o de diferentes patologías agudas, realizado por un especialista de acuerdo con los parámetros convencionales. Posteriormente se comparó este resultado con la metodología matemática, calculando la especificidad y la sensibilidad. Dichas medidas se realizaron a través de una clasificación binaria donde los verdaderos positivos (VP) son el número de pacientes diagnosticados dentro de los límites de anormalidad y que se encuentran dentro de los valores matemáticos correspondientes al mismo diagnóstico, falsos positivos (FP) son el número de Holters que matemáticamente se comportan como estudios dentro de la anormalidad y cuyo diagnóstico clínico es normal, falsos negativos (FN) son el número de Holters diagnosticados clínicamente como normales pero cuyos valores matemáticos se corresponden con casos patológicos con enfermedades agudas y finalmente los verdaderos negativos (VN) son definidos como el número de Holters diagnosticados clínicamente como normales y cuyos valores matemáticos también se corresponden con normalidad.

Con el objetivo de evaluar la concordancia entre los valores físicos-matemáticos y el diagnóstico clínico convencional se calculará el coeficiente Kappa a través de la siguiente fórmula:

$$
K=\frac{C_{o}-C a}{T_{o}-C a}
$$


Donde:

Co: número de concordancias observadas, es decir, número de pacientes con el mismo diagnóstico de acuerdo con la nueva metodología propuesta y con el Gold Standard.

To: totalidad de observaciones, es decir, la totalidad de casos normales y con enfermedades agudas.

Ca: Concordancias atribuibles al azar, que se calculan de acuerdo con la siguiente fórmula:

$$
C a=\left[\left(f_{1} C_{1}\right) / T o\right]+\left[\left(f_{2} C_{2}\right) / T o\right]
$$

Donde $\mathrm{f} 1$ es el número de pacientes que presentan valores matemáticos dentro de los límites de normalidad, $\mathrm{C} 1$ es el número de pacientes diagnosticados clínicamente dentro de la normalidad, $\mathrm{f} 2$ es el número de pacientes que presentan valores matemáticos asociados a enfermedades agudas, C2 es el número de pacientes diagnosticados clínicamente con enfermedades agudas y To es el número total de casos normales y con enfermedades agudas.

\section{Resultados}

De los 100 holters patológicos, 35 presentaron enfermedades agudas de acuerdo con las conclusiones del holter, ver tabla 1. Los rangos de las frecuencias cardiacas de los 115 holters variaron entre 45 y 190 lat/min, para un total de 28 rangos, ver tabla 2 , y los valores de probabilidad de estos rangos variaron entre 0 y 0,536 , ver tabla 3 . Los holter presentaron entre 6 y 21 rangos de frecuencias en total, presentándose entre 13 y 21 para los normales y entre 6 y 17 rangos en holters enfermos, de los cuales los casos con patologías agudas presentaron entre 6 y 13 rangos.

Se obtuvieron valores en la diferencia entre los rangos de las frecuencias más probables que oscilaron entre 5 y 25 , encontrándose entre 5 y 15 para los normales y entre 5 y 25 para los enfermos, de los cuales los que presentaron enfermedad aguda se encontraron entre 5 y 20.

El número total de latidos por hora tomó valores contenidos dentro de 30 rangos, entre 2.000 y 9.250 lat/hora. Las probabilidades halladas para el número total de latidos por hora variaron entre 0 y 0,696; la probabilidad máxima para el total de latidos osciló entre 0,130 y 0,696 , encontrando que en los holter normales solo se presentó un caso con probabilidad menor o igual a 0,217 y 3 con probabilidad mayor o igual a 0,304 , mientras que en los Holters enfermos se encontraron 12 con probabilidad menor o igual a 0,217 y 49 con probabilidad mayor o igual a 0,304 , de los cuales, ningún caso de enfermedad aguda presentó valores con probabilidad menor o igual a 0,217 , mientras que todos presentaron valores mayores o iguales a 0,304 .

Se presentaron 20 holters con número de latidos inferior a 3.000 y 14 con número de latidos superior a 6.250 , de los cuales 2 con número de latidos inferior a 3.000 se encontraron en el grupo dentro de los límites normales y los valores restantes se encontraron en el grupo de Holters enfermos. Dentro de estos últimos, 5 de los Holter con enfermedad aguda presentaron un número de latidos menor a 3.000 y ninguno presentó valores mayores a 6.250 .

La suma de las probabilidades de las dos frecuencias más probables osciló entre 0,203 y 0,652, presentando valores entre 0,203 y 0,379 para los normales y entre 0,203 y 0,652 para los enfermos, de los cuales los Holters con patologías agudas presentaron valores entre 0,333 y 0,652 .

La tabla 1 presenta la edad, indicaciones y diagnósticos convencionales de 10 holter normales y 10 con enfermedad aguda. La Tabla 4 presenta los valores matemáticos analizados para la determinación del diagnóstico para estos mismos casos. 
Tabla 1. Edad, indicaciones y diagnóstico de 20 Holters evaluados de acuerdo con los parámetros convencionales, escogidos dentro de los 115 estudiados. Los 10 primeros corresponden a estudios dentro de los límites normales y los 10 últimos corresponden a enfermedad aguda.

\begin{tabular}{|c|c|c|c|}
\hline No. & Edad & INDICACIONES & Resultados \\
\hline 5 & 46 & Palpitaciones & Estudio dentro de límites normales \\
\hline 12 & 42 & Cefalea, vértigo & Estudio dentro de límites normales \\
\hline 13 & 27 & Estudio de Síncope & Estudio dentro de límites normales \\
\hline 27 & 51 & Vértigo & Estudio dentro de límites normales \\
\hline 29 & 52 & Taquicardia & Estudio dentro de límites normales \\
\hline 33 & 32 & Taquicardia & Estudio dentro de límites normales \\
\hline 57 & 56 & Palpitaciones & Estudio dentro de límites normales \\
\hline 58 & 36 & Estudio de Síncope & Estudio dentro de límites normales \\
\hline 67 & 22 & Fatiga & Estudio dentro de límites normales \\
\hline 83 & 31 & Taquicardia & Estudio dentro de límites normales \\
\hline 1 & 66 & Arritmia no especificada & Extrasistolia supraventricular ocasional \\
\hline 7 & 65 & Bradicardia & $\begin{array}{l}\text { Pausas sinusales diurnas y nocturnas con maxima duración de } \\
\qquad 2,4 \text { segundos }\end{array}$ \\
\hline 16 & 54 & Estudio de control & $\begin{array}{l}\text { Extrasistolia supraventricular conducida ocasional. } \\
\text { Extrasistolia ventricular monomórfica frecuente. } \\
\text { Disminución de la variabilidad de la frecuencia cardiaca }\end{array}$ \\
\hline 23 & 49 & $\begin{array}{l}\text { Paciente con arritmia previa, } \\
\text { estudio de control. }\end{array}$ & $\begin{array}{l}\text { Bloqueo de rama izquierda intermitente dependiente } \\
\text { de incremento en frecuencia cardiaca }\end{array}$ \\
\hline 25 & 55 & Fibrilación auricular & $\begin{array}{l}\text { Extrasistolia ventricular y supraventricular monomórfica } \\
\text { frecuente sin fenómenos repetitivos }\end{array}$ \\
\hline 38 & 50 & Fibrilación auricular & $\begin{array}{c}\text { Extrasistolia ventricular con dos morfologías, frecuente con una } \\
\text { salva de taquicardia ventricular polimórfica no sostenida. } \\
\text { Variabilidad de la frecuencia cardiaca moderada } \\
\text { a levemente disminuida }\end{array}$ \\
\hline 45 & 59 & Sospecha de disfunción nodal & $\begin{array}{l}\text { Fibrilación auricular con respuesta ventricular lenta. Trastorno de } \\
\text { conducción intraventricular. Extrasistolia ventricular muy frecuen- } \\
\text { te de al menos dos morfologías con bigeminismo, dupletas y un } \\
\text { episodio de taquicardia ventricular no sostenida }\end{array}$ \\
\hline 65 & 46 & Hipertiroidismo & $\begin{array}{c}\text { Ectopia auricular infrecuente con una salva corta y aislada de } \\
\text { taquicardia auricular (sugestiva de reentrada perisinusal) }\end{array}$ \\
\hline 70 & 90 & Fibrilación auricular & $\begin{array}{c}\text { Extrasístoles auriculares infrecuentes. Taquicardia auricular no } \\
\text { sostenida. Moderada disminución de la variabilidad } \\
\text { de la frecuencia cardiaca }\end{array}$ \\
\hline 114 & 81 & Accidente cerebrovascular cardioembólico & $\begin{array}{c}\text { La conducción auriculoventricular e interventricular normal, no } \\
\text { se observa arritmias supraventriculares. Extrasistolia } \\
\text { ventricular monomorfica frecuente con bigeminismo, } \\
\text { adecuada variabilidad de la frecuencia cardiaca }\end{array}$ \\
\hline
\end{tabular}


Rodríguez J., Correa C, Prieto P., Bernal P., Forero G., Salazar G., Vitery S., Alvarez L., Puerta G.

\begin{tabular}{|c|c|c|c|c|c|c|c|c|c|c|c|c|c|c|c|c|c|c|c|c|c|c|c|}
\hline$\Xi$ & & & & & & \begin{tabular}{|l}
$\infty$ \\
0 \\
0 \\
0 \\
0
\end{tabular} & 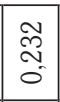 & 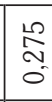 & $\begin{array}{l}\overrightarrow{5} \\
0 \\
0\end{array}$ & $\begin{array}{l}0 \\
\stackrel{0}{0} \\
0\end{array}$ & \begin{tabular}{|l|} 
\\
0 \\
0 \\
0 \\
0
\end{tabular} & 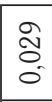 & & & & & & & & & & & 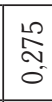 \\
\hline$R$ & & & $\begin{array}{l}\text { J } \\
\text { J } \\
0\end{array}$ & 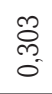 & $\begin{array}{l}\mathcal{Z} \\
\mathbb{N} \\
O\end{array}$ & $\begin{array}{l}0 \\
\text { ? } \\
0\end{array}$ & \begin{tabular}{|l}
0 \\
0 \\
0 \\
0
\end{tabular} & & $\begin{array}{l}0 \\
0 \\
0 \\
0\end{array}$ & & & & & & & & & & & & & & \begin{tabular}{|l}
0 \\
0 \\
0 \\
0 \\
0
\end{tabular} \\
\hline$\not 8$ & & & & $\begin{array}{l}\text { H } \\
0 \\
0\end{array}$ & $\begin{array}{l}\stackrel{0}{\circ} \\
\text { O. }\end{array}$ & $\begin{array}{l}\vec{\sigma} \\
\vec{\sigma}\end{array}$ & $\mid \begin{array}{l}\infty \\
0 \\
0 \\
0\end{array}$ & $\begin{array}{l}\tilde{N} \\
0 \\
0\end{array}$ & $\begin{array}{l}\text { S } \\
0 \\
0\end{array}$ & 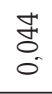 & $\begin{array}{l}\overrightarrow{0} \\
\dot{0} \\
0 \\
0\end{array}$ & $\begin{array}{l}\text { G } \\
\text { Oे } \\
0\end{array}$ & $\begin{array}{l}\text { बे } \\
\text { : }\end{array}$ & $\begin{array}{c}0 \\
\tilde{O} \\
0\end{array}$ & $\begin{array}{l}0 \\
\stackrel{8}{0} \\
0\end{array}$ & & \begin{tabular}{|l}
2 \\
0 \\
0 \\
0
\end{tabular} & & & & & & 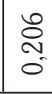 \\
\hline$\stackrel{8}{9}$ & $\begin{array}{l}0 \\
0 \\
0 \\
0\end{array}$ & 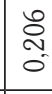 & $\begin{array}{l}\text { ôे } \\
\text { of } \\
\end{array}$ & $\begin{array}{l}\frac{1}{2} \\
0 \\
0 \\
\end{array}$ & \begin{tabular}{|l}
0 \\
$\hat{0}$ \\
0
\end{tabular} & $\begin{array}{l}2 \\
0 \\
0 \\
0\end{array}$ & $\begin{array}{l}\infty \\
0 \\
0 \\
0\end{array}$ & : & & & $\begin{array}{l}0 \\
0 \\
0 \\
0\end{array}$ & & & & & & & & & & & & $\begin{array}{l}\text { ôे } \\
\text { ô. } \\
\end{array}$ \\
\hline$\stackrel{\infty}{\infty}$ & & & & & $\begin{array}{l}\text { So } \\
0 \\
0\end{array}$ & $\mid \begin{array}{l}1 \\
0 \\
0 \\
0\end{array}$ & 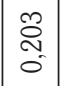 & $\begin{array}{l}\text { जे } \\
\text { O }\end{array}$ & $\begin{array}{l}\text { Oे } \\
\text { o. }\end{array}$ & 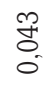 & $\left|\begin{array}{l}\infty \\
0 \\
0 \\
0\end{array}\right|$ & $\begin{array}{l}\vec{H} \\
0 \\
0\end{array}$ & & & & & & & & & & & $\begin{array}{l}\text { సે } \\
\text { O }\end{array}$ \\
\hline$\stackrel{20}{2}$ & & & & & $\begin{array}{l}2 \\
0 \\
0 \\
0\end{array}$ & & $\begin{array}{c}0 \\
0 \\
0 \\
0\end{array}$ & $\begin{array}{l}\infty \\
\stackrel{0}{0} \\
0\end{array}$ & $\begin{array}{l}\vec{J} \\
0 \\
0\end{array}$ & $\begin{array}{l}0 \\
0 \\
0 \\
0\end{array}$ & $\mid$\begin{tabular}{c}
$n$ \\
\multirow{2}{0}{} \\
0 \\
0
\end{tabular} & 总 & $\begin{array}{l}\stackrel{2}{0} \\
0 \\
0\end{array}$ & $\left|\begin{array}{l}2 \\
0 \\
0 \\
0\end{array}\right|$ & $\mid \begin{array}{c}0 \\
0 \\
0 \\
0\end{array}$ & 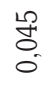 & \begin{tabular}{|l}
2 \\
0 \\
0 \\
0
\end{tabular} & & & & & & $\mid \begin{array}{l}\infty \\
0 \\
0\end{array}$ \\
\hline$\tilde{N}$ & \begin{tabular}{|l}
0 \\
0 \\
0 \\
0
\end{tabular} & $\begin{array}{l}+ \\
0 \\
0\end{array}$ & 음 & $\begin{array}{l}\overrightarrow{0} \\
0 \\
0\end{array}$ & $\mid \begin{array}{l}\hat{a} \\
0 \\
0\end{array}$ & 告 & $\mid \begin{array}{l}\infty \\
0 \\
0 \\
0\end{array}$ & $\begin{array}{l}\tilde{N} \\
0 \\
0 \\
0\end{array}$ & & & & & & & & & & & & & & & $\begin{array}{l}\text { ò } \\
\text { o. }\end{array}$ \\
\hline 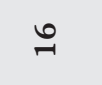 & & & & & & & $\begin{array}{l}0 \\
0 \\
0 \\
0\end{array}$ & $\begin{array}{l}\infty \\
\text { òd } \\
0 \\
0\end{array}$ & $\begin{array}{l}\text { Oे } \\
\text { ô } \\
0\end{array}$ & $\begin{array}{l}\vec{I} \\
\text { ज. }\end{array}$ & \begin{tabular}{|l}
0 \\
0 \\
0 \\
0
\end{tabular} & $\begin{array}{l}0 \\
0 \\
0 \\
0\end{array}$ & $\begin{array}{l}\stackrel{2}{0} \\
\stackrel{0}{\circ}\end{array}$ & & & & & & & & & & \begin{tabular}{|l}
$\infty$ \\
ơd \\
0.0
\end{tabular} \\
\hline r & \begin{tabular}{|c|}
2 \\
$\tilde{o}$ \\
0
\end{tabular} & $\begin{array}{l}\infty \\
0 \\
0 \\
0 \\
0\end{array}$ & 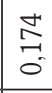 & 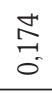 & $\begin{array}{l}\infty \\
\stackrel{\infty}{0} \\
0\end{array}$ & 孚 & $\begin{array}{l}2 \\
\tilde{O} \\
0\end{array}$ & $\begin{array}{l}\infty \\
\stackrel{\infty}{8} \\
0 \\
0\end{array}$ & $\begin{array}{l}1 \\
0 \\
0 \\
0\end{array}$ & $\begin{array}{l}\vec{H} \\
\dot{0} \\
0\end{array}$ & 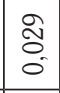 & $\begin{array}{l}\vec{H} \\
0 \\
0\end{array}$ & & & & & & & & & & & $\begin{array}{l}\infty \\
0 \\
0 \\
0\end{array}$ \\
\hline- & $\begin{array}{l}0 \\
\vdots \\
0\end{array}$ & 总 & $\begin{array}{l}\text { 今. } \\
\text { ô } \\
0\end{array}$ & $\begin{array}{l}0 \\
\text { : } \\
0\end{array}$ & $\begin{array}{l}\tilde{N} \\
\tilde{O} \\
0\end{array}$ & $\begin{array}{l}\overrightarrow{0} \\
0 \\
0\end{array}$ & & $\begin{array}{l}\infty \\
\ddot{0} \\
0 \\
0\end{array}$ & 今े & $\begin{array}{l}\infty \\
0 \\
0 \\
0\end{array}$ & \begin{tabular}{|l}
$\infty$ \\
0 \\
0 \\
0 \\
0
\end{tabular} & & $\begin{array}{l}\overrightarrow{0} \\
0 \\
0\end{array}$ & & & $\begin{array}{l}\text { :े } \\
\text { : }\end{array}$ & & & & & & & $\begin{array}{l}\text { Oे } \\
\text { âj }\end{array}$ \\
\hline$\infty$ & & & & $\begin{array}{l}\infty \\
0 \\
0 \\
0\end{array}$ & \begin{tabular}{|l} 
के \\
0 \\
0
\end{tabular} & $\begin{array}{l}\vec{\sigma} \\
0 \\
0\end{array}$ & $\begin{array}{l}\overrightarrow{5} \\
\overrightarrow{0} \\
0\end{array}$ & $\begin{array}{l}0 \\
\ddot{0} \\
0\end{array}$ & $\begin{array}{l}\frac{2}{9} \\
0 \\
0\end{array}$ & $\begin{array}{l}\tilde{N} \\
\tilde{0} \\
0 \\
0\end{array}$ & $\begin{array}{l}0 \\
0 \\
0 \\
0\end{array}$ & $\begin{array}{l}\infty \\
0 \\
0 \\
0 \\
0\end{array}$ & $\begin{array}{l}\text { के } \\
\text { : }\end{array}$ & $\begin{array}{l}0 \\
\tilde{0} \\
0 \\
0\end{array}$ & $\begin{array}{l}0 \\
\tilde{0} \\
0 \\
0\end{array}$ & $\begin{array}{l}\text { के } \\
0 \\
0\end{array}$ & $\begin{array}{l}\vec{J} \\
0 \\
0\end{array}$ & & $\begin{array}{c}\text { aे } \\
\dot{0}\end{array}$ & $\begin{array}{l}\text { : } \\
\text { : } \\
0\end{array}$ & & & 悉 \\
\hline to & $\begin{array}{l}\vec{z} \\
0 \\
0 \\
0\end{array}$ & 兽 & 党 & $\begin{array}{l}\infty \\
0 \\
0 \\
0\end{array}$ & $\begin{array}{l}\infty \\
0 \\
0 \\
0\end{array}$ & $\begin{array}{l}\frac{\pi}{ \pm} \\
0 \\
0\end{array}$ & $\begin{array}{l}\infty \\
0 \\
0 \\
0\end{array}$ & $\begin{array}{l}\infty \\
\ddot{8} \\
0 \\
0\end{array}$ & $\begin{array}{l}N \\
\tilde{O} \\
0 \\
0\end{array}$ & $\begin{array}{l}\text { के } \\
\text { o. }\end{array}$ & $\begin{array}{l}\overrightarrow{0} \\
\overrightarrow{0} \\
0\end{array}$ & $\begin{array}{l}\infty \\
0 \\
0 \\
0\end{array}$ & $\begin{array}{l}\text { के } \\
0 \\
0\end{array}$ & $\mid \begin{array}{l}\overrightarrow{0} \\
0 \\
0\end{array}$ & $\begin{array}{l}\vec{a} \\
0 \\
0\end{array}$ & $\begin{array}{l}\vec{\Delta} \\
0_{0}\end{array}$ & & $\begin{array}{l}\vec{\Delta} \\
\overrightarrow{0} \\
0 \\
0\end{array}$ & & $\begin{array}{l}\vec{\Delta} \\
\overrightarrow{0} \\
0\end{array}$ & & $\begin{array}{l}\vec{\Delta} \\
\overrightarrow{0} \\
0 \\
0\end{array}$ & $\begin{array}{l}\frac{H}{2} \\
\frac{0}{0}\end{array}$ \\
\hline$\stackrel{\infty}{\circ}$ & $\begin{array}{l}\infty \\
0 \\
0 \\
0\end{array}$ & \begin{tabular}{|l}
$\mid \hat{o g}$ \\
0 \\
0
\end{tabular} & $\begin{array}{l}\infty \\
0 \\
0 \\
0\end{array}$ & $\begin{array}{l}\overrightarrow{5} \\
\stackrel{0}{0}\end{array}$ & $\begin{array}{l}\overrightarrow{0} \\
0 \\
0\end{array}$ & $\begin{array}{l}\overrightarrow{0} \\
0 \\
0\end{array}$ & $\begin{array}{l}1 \\
0 \\
0 \\
0\end{array}$ & $\begin{array}{c}\overrightarrow{0} \\
0 \\
0\end{array}$ & $\begin{array}{l}1 \\
0 \\
0 \\
0\end{array}$ & $\begin{array}{l}\text { aे } \\
\text { Oे. }\end{array}$ & $\begin{array}{l}\vec{H} \\
0 \\
0 \\
0\end{array}$ & $\begin{array}{l}\infty \\
0 \\
0 \\
0\end{array}$ & $\begin{array}{l}\text { O্ } \\
\stackrel{0}{0}\end{array}$ & $\begin{array}{l}\overrightarrow{0} \\
0 \\
0 \\
0\end{array}$ & $\mid \begin{array}{c}m \\
0 \\
0 \\
0\end{array}$ & $\begin{array}{l}\overrightarrow{0} \\
\text { : }\end{array}$ & & & & & & & 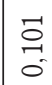 \\
\hline is & & $\begin{array}{l}\overrightarrow{0} \\
\dot{0} \\
0\end{array}$ & $\begin{array}{l}\overrightarrow{0} \\
0 \\
0\end{array}$ & 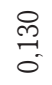 & $\begin{array}{l}0 \\
\because \\
0\end{array}$ & 悉 & \begin{tabular}{|l}
$\infty$ \\
0 \\
0 \\
0
\end{tabular} & $\begin{array}{l}\tilde{N} \\
\tilde{D} \\
0\end{array}$ & $\begin{array}{l}\overrightarrow{0} \\
0 \\
0\end{array}$ & $\begin{array}{l}\text { So } \\
0 \\
0\end{array}$ & \begin{tabular}{|l|}
$\hat{\alpha}$ \\
0 \\
0 \\
0
\end{tabular} & वे & $\begin{array}{l}\overrightarrow{0} \\
0 \\
0\end{array}$ & $\begin{array}{c}\overrightarrow{0} \\
0 \\
0 \\
0\end{array}$ & $\mid \begin{array}{c}2 \\
\dot{0} \\
0\end{array}$ & & & & & & & & $\mid \begin{array}{l}2 \\
\vdots \\
0\end{array}$ \\
\hline$\ddot{m}$ & & & $\begin{array}{l}2 \\
0 \\
0 \\
0\end{array}$ & $\begin{array}{l}\text { 웅 } \\
0\end{array}$ & $\begin{array}{l}0 \\
\stackrel{0}{0} \\
0\end{array}$ & 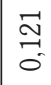 & 惫 & $\begin{array}{l}\vec{I} \\
\tilde{O}\end{array}$ & $\begin{array}{l}\tilde{N} \\
\text { O. }\end{array}$ & $\begin{array}{l}0 \\
\text { : } \\
0\end{array}$ & $\begin{array}{l}\vec{b} \\
0 \\
0\end{array}$ & 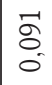 & $\begin{array}{l}\stackrel{2}{0} \\
0 \\
0\end{array}$ & $\mid \begin{array}{l}2 \\
0 \\
0 \\
0\end{array}$ & & & & $\begin{array}{l}\frac{2}{2} \\
0 \\
0 \\
0\end{array}$ & & & & & $\begin{array}{l}\tilde{\omega} \\
0 \\
0\end{array}$ \\
\hline ลิ & & & $\begin{array}{l}\tilde{N} \\
0 \\
0 \\
0\end{array}$ & $\begin{array}{l}\hat{y} \\
\text { c. }\end{array}$ & 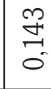 & $\begin{array}{l}\frac{\mu}{2} \\
0 \\
0\end{array}$ & 音 & $\begin{array}{l}\infty \\
0 \\
0 \\
0\end{array}$ & $\begin{array}{l}\infty \\
0 \\
0 \\
0\end{array}$ & $\begin{array}{l}\text { ôे } \\
\text { o. }\end{array}$ & $\mid \begin{array}{l}0 \\
0 \\
0 \\
0\end{array}$ & $\begin{array}{l}\infty \\
0 \\
0 \\
0 \\
0\end{array}$ & $\begin{array}{l}\text { 我 } \\
0 \\
0\end{array}$ & $\left|\begin{array}{c}\tilde{N} \\
0 \\
0 \\
0\end{array}\right|$ & & & & $\begin{array}{l}\tilde{\tilde{O}} \\
0 \\
0 \\
0\end{array}$ & & $\begin{array}{l}0 \\
\stackrel{0}{0} \\
0 \\
0\end{array}$ & & & $\begin{array}{l}\stackrel{2}{2} \\
0 \\
0\end{array}$ \\
\hline$\hat{N}$ & & & $\begin{array}{l}0 \\
0 \\
0 \\
0\end{array}$ & $\begin{array}{l}\text { 令 } \\
\text { o. }\end{array}$ & $\mid \begin{array}{l}\hat{\sigma} \\
\dot{0} \\
0\end{array}$ & 总 & 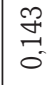 & $\begin{array}{l}\bar{N} \\
0 \\
0\end{array}$ & $\begin{array}{l}\tilde{a} \\
0 \\
0\end{array}$ & $\begin{array}{l}\text { Oू } \\
: \\
0 .\end{array}$ & $\begin{array}{l}\hat{\sigma} \\
0 \\
0\end{array}$ & $\mid \begin{array}{l}\infty \\
0 \\
0 \\
0 \\
0\end{array}$ & $\begin{array}{l}\tilde{O} \\
\text { Oे } \\
0\end{array}$ & $\mid \begin{array}{l}\tilde{N} \\
0 \\
0 \\
0\end{array}$ & & & $\begin{array}{l}0 \\
0 \\
0 \\
0\end{array}$ & & & & & & $\mid \begin{array}{l}\stackrel{3}{a} \\
0\end{array}$ \\
\hline$\stackrel{m}{\rightarrow}$ & & & & & $\begin{array}{l}1 \\
0 \\
0 \\
0\end{array}$ & 总 & $\mid \begin{array}{l}\infty \\
0 \\
0 \\
0\end{array}$ & 音 & $\begin{array}{l}\infty \\
0 \\
0 \\
0\end{array}$ & $\begin{array}{l}\infty \\
\stackrel{0}{0} \\
0\end{array}$ & $\begin{array}{l}0 \\
0 \\
0 \\
0\end{array}$ & $\begin{array}{l}0 \\
0 \\
0 \\
0\end{array}$ & $\begin{array}{l}\stackrel{2}{2} \\
0 \\
0\end{array}$ & $\mid \begin{array}{l}2 \\
0 \\
0 \\
0\end{array}$ & $\mid \begin{array}{c}2 \\
0 \\
0 \\
0\end{array}$ & $\begin{array}{l}\stackrel{2}{0} \\
0 \\
0\end{array}$ & & & $\begin{array}{l}2 \\
0 \\
0 \\
0\end{array}$ & $\begin{array}{l}2 \\
0 \\
0 \\
0 \\
0\end{array}$ & & & $\mid \begin{array}{l}\infty \\
0 \\
0\end{array}$ \\
\hline$\approx$ & & $\mid \begin{array}{l}\tilde{N} \\
0 \\
0\end{array}$ & 党 & $\begin{array}{l}\text { 足 } \\
\text { o. }\end{array}$ & $\begin{array}{l}\vec{a} \\
\dot{0}\end{array}$ & 总 & \begin{tabular}{|l}
$\infty$ \\
0 \\
0 \\
0 \\
0
\end{tabular} & $\begin{array}{l}\tilde{N} \\
0 \\
0\end{array}$ & $\begin{array}{l}0 \\
0 \\
0 \\
0\end{array}$ & $\begin{array}{l}\tilde{N} \\
\tilde{0} \\
0\end{array}$ & $\begin{array}{l}\mathcal{O} \\
\text { Oे } \\
\text { O. }\end{array}$ & $\mid \begin{array}{l}\infty \\
0 \\
0 \\
0 \\
0\end{array}$ & $\begin{array}{l}\vec{H} \\
\text { 品 }\end{array}$ & $\left|\begin{array}{l}\infty \\
\ddot{8} \\
0 \\
0\end{array}\right|$ & $\mid \begin{array}{c}0 \\
\tilde{O} \\
0\end{array}$ & $\begin{array}{l}\text { के } \\
0 \\
0\end{array}$ & $\begin{array}{l}\vec{J} \\
0 \\
0\end{array}$ & & & & & & $\begin{array}{l}\text { 㔽 } \\
0\end{array}$ \\
\hline مـ & & & & $\begin{array}{l}\vec{H} \\
0 \\
0 \\
0\end{array}$ & $\begin{array}{l}\infty \\
0 \\
0 \\
0 \\
0\end{array}$ & $\begin{array}{l}\overrightarrow{\vec{b}} \\
0\end{array}$ & 孚 & $\begin{array}{l}\stackrel{0}{0} \\
\text { O. }\end{array}$ & $\begin{array}{l}\infty \\
0 \\
0 \\
0 \\
0\end{array}$ & $\begin{array}{l}0 \\
\text { : } \\
0\end{array}$ & $\begin{array}{l}0 \\
\text { : } \\
0\end{array}$ & $\begin{array}{l}\infty \\
\stackrel{0}{0} \\
0\end{array}$ & \begin{tabular}{l} 
\%े \\
\multirow{0}{0}{}
\end{tabular} & $\begin{array}{c}c \\
\stackrel{o}{0} \\
\dot{0}\end{array}$ & 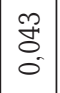 & $\begin{array}{l}\overrightarrow{0} \\
\dot{0}\end{array}$ & $\begin{array}{l}\vec{\sigma} \\
0 \\
0\end{array}$ & & & & & & $\begin{array}{l}\text { Oे } \\
\text { o. } \\
0\end{array}$ \\
\hline 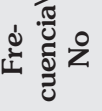 & \& & in & 㖞 & 8 & 88 & 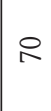 & $\stackrel{2}{2}$ & $\infty$ & $\infty$ & 8 & ณ & $\stackrel{-1}{\circ}$ & 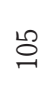 & 윽 & $\stackrel{\Omega}{\exists}$ & త్తి & $\underset{\sim}{\stackrel{\sim}{\sim}}$ & 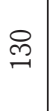 & $\stackrel{\stackrel{m}{g}}{\sim}$ & gi & 孚 & 욤 & \begin{tabular}{|l} 
訔 \\
离
\end{tabular} \\
\hline
\end{tabular}




\begin{tabular}{|c|c|c|c|c|c|c|c|c|c|c|c|c|c|c|c|c|}
\hline$\underset{\Xi}{\Xi}$ & & & & & & & & 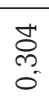 & $\begin{array}{l}10 \\
0 \\
0 \\
0\end{array}$ & $\begin{array}{l}\stackrel{9}{0} \\
\stackrel{0}{0}\end{array}$ & & & & & & $\begin{array}{l}10 \\
\text { L } \\
0 \\
0\end{array}$ \\
\hline$\stackrel{R}{R}$ & & & $\begin{array}{l}8 \\
8 \\
0 \\
0\end{array}$ & $\begin{array}{l}8 \\
0 \\
0 \\
0\end{array}$ & $\begin{array}{l}\infty \\
\infty \\
0 \\
0\end{array}$ & $\begin{array}{l}\stackrel{2}{N} \\
0 \\
0\end{array}$ & & & & & & & & & & $\begin{array}{l}8 \\
8 \\
0 \\
0\end{array}$ \\
\hline 12 & & & & & & $\begin{array}{l}\overrightarrow{\mathcal{L}} \\
\text { o. }\end{array}$ & 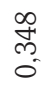 & $\begin{array}{l}\overrightarrow{\mathrm{S}} \\
\text { on }\end{array}$ & ঙ্ণ & $\begin{array}{l}\infty \\
\dot{0} \\
0\end{array}$ & & & & & & $\begin{array}{l}\text { के } \\
\text { ஸे } \\
0\end{array}$ \\
\hline$\stackrel{2}{\&}$ & & $\begin{array}{l}\stackrel{F}{ \pm} \\
\stackrel{0}{0}\end{array}$ & ָे & 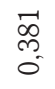 & \begin{tabular}{l}
$\infty$ \\
\multirow{0}{*}{} \\
0
\end{tabular} & & & & & & & & & & & Iิ \\
\hline$\stackrel{\infty}{\infty}$ & & & & & & & $\begin{array}{l}\text { O্ষ } \\
\stackrel{0}{\circ}\end{array}$ & 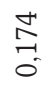 & $\begin{array}{l}\text { ¿े } \\
\text { m. } \\
\text { ó }\end{array}$ & 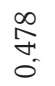 & & & & & & 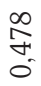 \\
\hline$\stackrel{\llcorner}{\sim}$ & & & & & & & & $\begin{array}{l}\vec{\sigma} \\
0 \\
0\end{array}$ & $\begin{array}{c}\infty \\
\stackrel{m}{m} \\
0\end{array}$ & $\begin{array}{l}\text { \& } \\
\text { वे } \\
0\end{array}$ & $\begin{array}{l}\overrightarrow{0} \\
0 \\
0\end{array}$ & $\begin{array}{l}\hat{N} \\
\text { N } \\
0\end{array}$ & $\begin{array}{l}\hat{N} \\
\text { N } \\
0\end{array}$ & & & $\begin{array}{l}\infty \\
\vec{m} \\
0\end{array}$ \\
\hline బ & 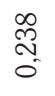 & $\begin{array}{l}\text { ָे } \\
\text { o }\end{array}$ & $\begin{array}{l}\infty \\
\stackrel{\sim}{3} \\
\stackrel{0}{0}\end{array}$ & $\begin{array}{l}20 \\
0 \\
0 \\
0\end{array}$ & & & & & & & & & & & & $\begin{array}{l}\text { ఫे } \\
\text { ธे }\end{array}$ \\
\hline$\stackrel{0}{\sim}$ & & & & & & & & & $\begin{array}{l}\text { oे } \\
\text { ó }\end{array}$ & 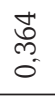 & $\begin{array}{l}0 \\
\stackrel{m}{0} \\
0\end{array}$ & $\begin{array}{l}\overrightarrow{0} \\
0 \\
0\end{array}$ & & & & $\begin{array}{l}\text { oे } \\
\text { ò } \\
\text { के }\end{array}$ \\
\hline - & & & $\begin{array}{l}\text { ô } \\
\text { - }\end{array}$ & $\begin{array}{l}\hat{N} \\
\text { ON }\end{array}$ & $\begin{array}{l}\text { ¿े } \\
\text { on }\end{array}$ & $\begin{array}{l}\tilde{N} \\
\text { Oे }\end{array}$ & $\begin{array}{l}\text { m } \\
\stackrel{0}{0}\end{array}$ & & & & & & & & & $\begin{array}{l}\text { d్ } \\
\text { o } \\
0\end{array}$ \\
\hline$\tau$ & $\begin{array}{l}\vec{D} \\
\text { ov } \\
0\end{array}$ & $\begin{array}{l}\vec{\sigma} \\
\text { O. } \\
\text { o. }\end{array}$ & $\begin{array}{l}\infty \\
\text { 心্ } \\
0 \\
0\end{array}$ & & & & & & & & & & & & & के \\
\hline$\infty$ & & & & & & $\begin{array}{l}\stackrel{0}{9} \\
\stackrel{0}{0}\end{array}$ & 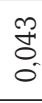 & $\begin{array}{l}0 \\
\text { m } \\
0\end{array}$ & 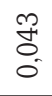 & $\begin{array}{l}\text { O্ } \\
\stackrel{0}{0}\end{array}$ & $\begin{array}{l}\stackrel{9}{0} \\
\text { 0. }\end{array}$ & $\begin{array}{l}\overrightarrow{0} \\
\text { O. } \\
0\end{array}$ & $\begin{array}{l}\stackrel{0}{0} \\
\stackrel{0}{0}\end{array}$ & 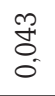 & & 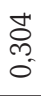 \\
\hline 6 & & & $\begin{array}{c}\text { ते } \\
\text { o } \\
0\end{array}$ & $\begin{array}{l}\text { m } \\
\text { o } \\
0\end{array}$ & $\begin{array}{l}\text { m } \\
\text { o } \\
0\end{array}$ & $\begin{array}{l}\text { o } \\
\text { : } \\
0\end{array}$ & $\begin{array}{l}\text { F } \\
0 \\
0\end{array}$ & $\begin{array}{l}\hat{0} \\
\text { : } \\
0\end{array}$ & $\begin{array}{l}\hat{0} \\
\text { o } \\
0\end{array}$ & $\begin{array}{l}\stackrel{+}{ \pm} \\
0 \\
\end{array}$ & & $\begin{array}{l}\text { F } \\
0 \\
0\end{array}$ & $\begin{array}{l}\text { o } \\
0 \\
0 \\
0\end{array}$ & & & $\begin{array}{l}\text { d } \\
\text { m } \\
0\end{array}$ \\
\hline$\stackrel{\infty}{\circ}$ & & & $\begin{array}{l}\stackrel{+}{N} \\
0 \\
0\end{array}$ & $\begin{array}{l}\hat{o} \\
0 \\
0\end{array}$ & $\begin{array}{l}\text { 品 } \\
\stackrel{0}{0}\end{array}$ & & $\begin{array}{l}\hat{0} \\
\tilde{O}_{0}^{\circ}\end{array}$ & $\begin{array}{l}0 \\
\text { on } \\
0\end{array}$ & $\begin{array}{l}0 \\
\text { m } \\
0\end{array}$ & $\begin{array}{l}\stackrel{+}{I} \\
\stackrel{0}{0}\end{array}$ & $\begin{array}{l}\mathscr{P} \\
\stackrel{0}{\circ}\end{array}$ & & $\begin{array}{l}\mathscr{P} \\
\stackrel{0}{0}\end{array}$ & & & $\frac{\text { 咅 }}{\text { - }}$ \\
\hline in & & & $\begin{array}{l}\hat{\alpha} \\
0 \\
0 \\
0\end{array}$ & $\begin{array}{l}\text { o } \\
\text { ? } \\
0\end{array}$ & $\begin{array}{l}\text { T⿱ } \\
\stackrel{5}{0}\end{array}$ & 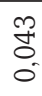 & \begin{tabular}{l}
$\vec{J}$ \\
\multirow{0}{0}{}
\end{tabular} & $\begin{array}{l}\hat{\alpha} \\
0 \\
0\end{array}$ & $\begin{array}{l}\text { m } \\
\text { Oे } \\
0\end{array}$ & $\begin{array}{l}\hat{0} \\
0 \\
0\end{array}$ & 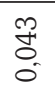 & $\begin{array}{l}\text { mat } \\
\stackrel{0}{0} \\
0^{\circ}\end{array}$ & & & & \begin{tabular}{l}
$\vec{D}$ \\
\multirow{N}{*}{} \\
0
\end{tabular} \\
\hline m & & & & & & \begin{tabular}{l} 
N \\
\multirow{N}{0}{} \\
0
\end{tabular} & $\begin{array}{l}\text { o } \\
\stackrel{0}{0}\end{array}$ & & $\begin{array}{l}0 \\
m \\
0 \\
0\end{array}$ & $\begin{array}{l}\text { Ñ } \\
\text { o }\end{array}$ & $\begin{array}{l}\infty \\
m \\
0 \\
0\end{array}$ & $\begin{array}{l}\vec{a} \\
0 \\
0\end{array}$ & & & & 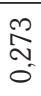 \\
\hline จิ & & & & & 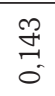 & 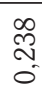 & $\begin{array}{l}20 \\
0 \\
0 \\
0\end{array}$ & $\begin{array}{l}\stackrel{\Re}{+} \\
0 \\
0\end{array}$ & $\begin{array}{l}\text { Lू } \\
0 \\
0\end{array}$ & & $\begin{array}{l}\stackrel{8}{0} \\
\stackrel{0}{0}\end{array}$ & $\begin{array}{l}\infty \\
\stackrel{\infty}{0} \\
0\end{array}$ & & & $\begin{array}{l}0 \\
\stackrel{\infty}{0} \\
0 \\
0\end{array}$ & 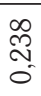 \\
\hline$\hat{\sim}$ & & & & & $\begin{array}{l}20 \\
8 \\
0 \\
0\end{array}$ & $\begin{array}{l}2 \\
2 \\
0 \\
0\end{array}$ & $\begin{array}{l}20 \\
8 \\
0 \\
0\end{array}$ & $\begin{array}{l}8 \\
\stackrel{2}{-} \\
0\end{array}$ & 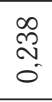 & $\begin{array}{l}20 \\
0 \\
0 \\
0\end{array}$ & 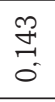 & 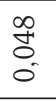 & & & & \begin{tabular}{l}
$\infty$ \\
\multirow{N}{*}{} \\
0 \\
0
\end{tabular} \\
\hline$\stackrel{m}{\rightarrow}$ & & & & & & & $\begin{array}{l}\bar{\sigma} \\
0 \\
0\end{array}$ & $\begin{array}{l}\stackrel{m}{N} \\
\text { o }\end{array}$ & & $\begin{array}{l}\text { N } \\
\text { O }\end{array}$ & $\begin{array}{l}\text { N } \\
\text { o } \\
\end{array}$ & & $\begin{array}{l}0 \\
\stackrel{m}{0} \\
0\end{array}$ & $\begin{array}{l}\text { Po } \\
0 \\
0\end{array}$ & & $\begin{array}{l}\text { N } \\
\text { N } \\
0\end{array}$ \\
\hline$\simeq$ & & & $\begin{array}{l}\hat{0} \\
\dot{0} \\
0\end{array}$ & $\begin{array}{l}\hat{N} \\
\text { o } \\
\end{array}$ & & $\begin{array}{l}\text { के } \\
\text { : }\end{array}$ & $\begin{array}{l}\mathscr{F} \\
\stackrel{5}{0} \\
0\end{array}$ & & $\begin{array}{l}\hat{0} \\
\dot{0} \\
0\end{array}$ & $\begin{array}{l}\text { సे } \\
\text { o. }\end{array}$ & $\begin{array}{l}\stackrel{+}{ \pm} \\
0 \\
0\end{array}$ & $\begin{array}{l}\hat{0} \\
0 \\
0\end{array}$ & & & & $\begin{array}{l}\text { जे } \\
\text { o }\end{array}$ \\
\hline مL & & & & & & & & 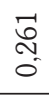 & $\begin{array}{l}\vec{D} \\
\text { o. }\end{array}$ & $\begin{array}{l}+ \\
\stackrel{+}{0} \\
0\end{array}$ & $\begin{array}{l}\stackrel{0}{0} \\
\stackrel{0}{0}\end{array}$ & $\begin{array}{l}\stackrel{m}{0} \\
0\end{array}$ & $\begin{array}{l}\text { : } \\
\vdots\end{array}$ & 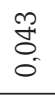 & & $\begin{array}{l}\vec{b} \\
\stackrel{s}{0}\end{array}$ \\
\hline 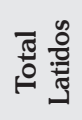 & 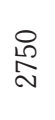 & ষ্ণ & $\begin{array}{l}\stackrel{\leftrightarrow}{心} \\
\text { }\end{array}$ & 总 & $\begin{array}{l}\stackrel{\circ}{\circ} \\
\text { nn }\end{array}$ & ষ & $\begin{array}{l}\stackrel{\leftrightarrow}{\sim} \\
\text { J }\end{array}$ & $\begin{array}{l}8 \\
\text { \& } \\
\text { S }\end{array}$ & 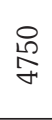 & : & $\begin{array}{l}\text { 岕 } \\
\text { ஸे }\end{array}$ & $\begin{array}{l}8 \\
\text { 员 } \\
\text { م. }\end{array}$ & 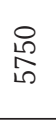 & 8 & 岕 & 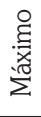 \\
\hline
\end{tabular}


Tabla 4. Valores matemáticos analizados para la determinación del diagnóstico en los 20 Holters escogidos dentro de los 115 estudiados. Los 10 primeros corresponden a estudios dentro de los límites normales y los 10 últimos corresponden a enfermedad aguda.

\begin{tabular}{|c|c|c|c|c|c|c|c|c|c|c|}
\hline \multirow{2}{*}{ No. } & \multirow{2}{*}{ Edad } & \multirow{2}{*}{$\begin{array}{l}\text { Dx } \\
\text { Conv. }\end{array}$} & \multirow{2}{*}{$\begin{array}{l}\text { Dx } \\
\text { Mat }\end{array}$} & \multirow{2}{*}{$\begin{array}{c}\text { No. } \\
\text { Rangos }\end{array}$} & \multirow{2}{*}{ Dif } & \multicolumn{2}{|c|}{ Máx prob No. Lat } & \multicolumn{2}{|c|}{ No. de Latidos } & \multirow{2}{*}{ Sum 2} \\
\hline & & & & & & $<=0,217$ & $>=0,304$ & $<3000$ & $>6250$ & \\
\hline 5 & 46 & $\mathrm{~N}$ & $e$ & 14 & 5 & & $\mathrm{x}$ & & & 0,347 \\
\hline 12 & 42 & $\mathrm{~N}$ & $\mathrm{~N}$ & 16 & 5 & & & & & 0,290 \\
\hline 13 & 27 & $\mathrm{~N}$ & $\mathrm{~N}$ & 14 & 5 & & & & & 0,318 \\
\hline 27 & 51 & $\mathrm{~N}$ & $\mathrm{~N}$ & 13 & 5 & & & & & 0,302 \\
\hline 29 & 52 & $\mathrm{~N}$ & $E$ & 14 & 15 & & & & & 0,317 \\
\hline 33 & 32 & $\mathrm{~N}$ & $\mathrm{~N}$ & 13 & 5 & & & & & 0,288 \\
\hline 57 & 56 & $\mathrm{~N}$ & $\mathrm{~N}$ & 14 & 5 & & & & & 0,275 \\
\hline 58 & 36 & $\mathrm{~N}$ & $e$ & 16 & 5 & $\mathrm{X}$ & & & & 0,203 \\
\hline 67 & 22 & $\mathrm{~N}$ & $\mathrm{~N}$ & 19 & 5 & & $\mathrm{x}$ & & & 0,304 \\
\hline 83 & 31 & $\mathrm{~N}$ & $e$ & 16 & 5 & & $\mathrm{x}$ & & & 0,261 \\
\hline 1 & 66 & $E$ & $E$ & 12 & 5 & & $\mathrm{x}$ & 12 & & 0,362 \\
\hline 7 & 65 & $E$ & $E$ & 12 & 15 & & $X$ & & & 0,362 \\
\hline 16 & 54 & $E$ & $E$ & 7 & 20 & & $x$ & & & 0,621 \\
\hline 23 & 49 & $E$ & $E$ & 8 & 10 & & $x$ & 10 & & 0,500 \\
\hline 25 & 55 & $E$ & $E$ & 12 & 10 & & $x$ & & & 0,333 \\
\hline 38 & 50 & $E$ & $E$ & 8 & 10 & & $x$ & & & 0,420 \\
\hline 45 & 59 & $E$ & $E$ & 9 & 5 & & $x$ & 1 & & 0,556 \\
\hline 65 & 46 & $E$ & $E$ & 13 & 20 & & $x$ & & & 0,397 \\
\hline 70 & 90 & $E$ & $E$ & 6 & 5 & & $x$ & & & 0,545 \\
\hline 114 & 81 & $E$ & $E$ & 7 & 5 & & $x$ & & & 0,507 \\
\hline
\end{tabular}

Dx. Conv: diagnóstico de normalidad o enfermedad de acuerdo con los parámetros convencionales. Dx Mat: diagnóstico matemático. N: Normalidad. E: Enfermedad. e: evolución entre normalidad y enfermedad. No. rangos: número de rangos de frecuencias cardiacas medidas. Dif: diferencia entre los rangos de las frecuencias cardiacas que presentaron los dos máximos valores de probabilidad. Máx prob No. Lat: máximo valor encontrado en las probabilidades del número total de latidos por hora. No. de latidos: frecuencia en que se presenta el número de latidos especificado. Sum 2: Suma de los dos valores de probabilidad más altos.

\section{Resultados estadísticos}

El análisis estadístico se realizó con los 15 holter diagnosticados como normales y los 35 con enfermedades agudas de acuerdo con los parámetros tradicionales, obtenidos de las conclusiones del holter y que fueron tomados como Gold Standard. Se determinó que el valor de sensibilidad fue del 100\%, mientras que la especificidad fue del 73,3\%, tabla 5 . El valor de la concordancia evaluado mediante el coeficiente Kappa entre el diagnóstico físico matemático y el diagnóstico clínico convencional, fue igual a 0,86.

Al observar los cuatro casos que fueron diagnosticados como normales desde los parámetros convencionales y cuyo diagnóstico matemático era diferente, se observó que tres casos habían sido diagnosticados matemáticamente en evolución a la enfermedad (holter No. 5, 58 y 83), y al observar las características de los holter se encontró que presentaban antecedentes de palpitaciones, taquicardia y síncope respectivamente; el primero y tercer caso presentaron valores de probabilidad máxima del número de latidos mayores a 0,304 y el segundo presentó una menor a 0,217. Además el primer caso presentó una suma de las dos probabilidades máximas de las frecuencias cardiacas mayor a 0,319, comportamiento que se presentó en todos los casos de enfermedad aguda. El cuarto Holter (No. 29) presentó un antecedente de taquicardia y presentó una diferencia de 15 entre los rangos de 
las frecuencias más probables, mientras que todo el resto de holter normales presentó diferencias de 5 , ver tablas 1 y 2 .

Tabla 5. Medidas de desempeño calculadas para los 15 holter normales y 35 con enfermedad aguda; comparación del método matemático de ayuda diagnóstica frente a la evaluación clínica, tomada como Gold-Standard.

\begin{tabular}{|c|c|c|}
\hline \multicolumn{3}{|c|}{ Clasificación Binaria } \\
\hline & Positivo & Negativo \\
\hline Positivo & 35 & 4 \\
\hline Negativo & 0 & 11 \\
\hline \multicolumn{2}{|c|}{ Medidas de desempeño } \\
\hline Sensibilidad = VP / (VP + FN) & $100 \%$ \\
\hline Especificidad = VN / (VN + FP) & $73,3 \%$ \\
\hline
\end{tabular}

\section{Discusión}

Este es el primer trabajo en el que se hace una confirmación de la aplicabilidad clínica de la metodología desarrollada para evaluación del holter con base en la teoría de la probabilidad, confirmando su utilidad como herramienta de ayuda diagnóstica. Se compararon los resultados obtenidos con los prototipos de normalidad y enfermedad del trabajo previo y se verificó la aplicabilidad de la metodología a cualquier caso particular, independientemente de la patología, intervenciones y de la edad para individuos mayores de 20 años, evidenciando que este método puede ser útil para determinar alteraciones que impliquen evolución hacia la enfermedad, y que no son detectadas desde los parámetros convencionales. La metodología desarrollada a partir de las leyes de la probabilidad, constituye una forma para la evaluación del holter con base en criterios sencillos y fácilmente aplicables. Tal como se había afirmado previamente, esta metodología puede ser útil para diseñar marcapasos basados en programaciones que mantengan rangos de valores apropiados de la frecuencia cardiaca, así como para la evaluación de la eficacia de intervenciones quirúrgicas o farmacológicas (9).

Los resultados del estudio estadístico evidencian la aplicabilidad clínica de esta metodología a la detección de patologías agudas, al presentar una sensibilidad del $100 \%$, mientras que la especificidad de $73,3 \%$, puede implicar la capacidad de detectar alteraciones leves no detectables mediante las metodologías convenciona- les, como se evidencia al observar los antecedentes $e$ indicaciones de los cuatro pacientes diagnosticados matemáticamente como enfermos o en evolución a la enfermedad y que presentaron un diagnóstico de normalidad desde los parámetros convencionales, quienes presentaron diversas sintomatologías o patologías previas, como palpitaciones, taquicardia o síncope que pueden implicar un funcionamiento que se está alejando de la normalidad, y que no puede evidenciarse desde las metodologías actualmente utilizadas. Adicionalmente el diagnóstico matemático indica que un número de latidos menor a 3.000 o mayor a 6.250 asociado a ciertos parámetros es indicador de evolución a la enfermedad o enfermedad, lo cual no es tenido en cuenta desde los parámetros tradicionales. Este hallazgo podría ser utilizado en la clínica como un indicador de pacientes que deberían ser mejor observados en el tiempo. La aplicación del coeficiente kappa, elegido para determinar la concordancia entre ambos diagnósticos independientemente de las concordancias atribuibles al azar, permitió establecer que la metodología matemática presenta una concordancia muy buena, al encontrarse por encima de 0,8 , que corresponde al rango más alto posible, pero como se aclaró previamente, el método matemático es más específico pues se aplica a casos individuales a diferencia del método convencional.

El interés actual en cardiología se centra en el estudio de la variabilidad (14-18), sin embargo desde esta perspectiva no es posible establecer diferencias para cada caso particular; este trabajo confirma que los métodos de diagnóstico basados en la variabilidad pueden ser mejorados, mediante métodos cuantitativos que evalúen la auto-organización del sistema, pues posibilitan diferenciar salud de enfermedad, así como distinguir diferenciaciones específicas de casos individuales clasificados convencionalmente de forma similar, siendo útil en la caracterización de estados de evolución entre normalidad y enfermedad.

Desde esta perspectiva han sido efectuados otros trabajos de tipo diagnóstico y predictivo para el Holter con base en teorías físicas y matemáticas. Por ejemplo, con base en la teoría de sistemas dinámicos, se desarrolló una nueva metodología de evaluación del holter con base en la ocupación espacial de atractores fractales en el espacio de Box- Counting (19), mediante la cual se diferencia enfermedad aguda de normalidad y enfermedad crónica. Esta metodología fue aplicada posterior-mente a 150 Holter para evaluar 
sus implicaciones clínicas, diferenciando normalidad de pacientes con IAM con una especificidad y una sensibilidad del 100\% (20). Siguiendo esta línea de investigación, se construyeron en el mapa de retardo atractores de tipo numérico para cada dinámica cardiaca durante 18 horas, evaluando la probabilidad de ocupación, entropía y sus proporciones para tres regiones de los atractores, estableciendo valores predictivos para normalidad y enfermedad que diferencian dinámicas cardiacas normales, crónicas y agudas, además de estados de evolución entre estos estados a partir de la probabilidad y las proporciones de la entropía (7). Esta metodología fue aplicada posteriormente al estudio de la evolución de la dinámica cardiaca de pacientes en la unidad de cuidados coronarios confirmando las predicciones diagnósticas realizadas. Por ejemplo, se encontró un caso que aunque es reportado clínicamente como asintomático, está en la Unidad de Cuidados Coronarios desde hace 15 días, cuya cuantificación desde la metodología matemática, se predijo que el sistema cardiaco estaba evolucionando hacia un estado más agudo aunque no se presentaban síntomas clínicos. De esta forma se evidencia que el uso de las predicciones físicas desarrolladas puede ser muy importante en la prevención a nivel clínico, pues permite evaluar de manera objetiva y cuantificada la evolución en el tiempo de un paciente, así como el impacto de intervenciones quirúrgicas y/o farmacológicas (8).

El uso de teorías físicas y matemáticas ha permitido también el desarrollo de una generalización teórica con la que se estableció un total 69.049 prototipos fractales arteriales posibles en el proceso de estenosis y reestenosis coronaria, con base el concepto de Armonía Matemática Intrínseca (21). En biología molecular por ejemplo fue posible desarrollar una teoría del fenómeno de unión de péptidos de malaria al glóbulo rojo mediante la cual se lograron predicciones respecto a los resultados experimentales con especificidad y sensibilidad del $95 \%$ y $92 \%$ respectivamente (22), mientras que en inmunología se logró una teoría predictiva del fenómeno de unión al HLA clase II basada en medidas de probabilidad, combinatoria y entropía (23). En el campo de la salud pública se desarrolló una metodología para la predicción de brotes de malaria en 820 municipios de Colombia en períodos de tres semanas, con un porcentaje de exactitud del 99.86\% (24). Del mismo modo se obtuvo un diagnóstico fractal de alteraciones preneolásicas y neoplásicas de células de cuello uterino con base en medidas de dimensión fractal (25). Estos resultados demuestran que los fenómenos médicos presentan formas de autoorganización fisicomatemática cuya determinación es útil en la práctica médica.

\section{Dedicación}

Este trabajo es dedicado a los profesores y estudiantes de la promoción de 1985, modalidad Ciencias y Matemáticas del INEM Francisco de Paula Santander de Kennedy, y en especial a José Raúl Rengifo Luna, Mejor Bachiller de la generación de 1978 del INEM de Kennedy, quien gracias a mi madre, fue mi profesor de física y matemáticas de toda la vida, y sin el cual nunca hubiera podido acercarme a la magia de la física y las matemáticas.

\section{Agradecimientos}

Agradecemos a la Universidad Militar Nueva Granada, especialmente al Dr. José Ricardo Cure Hakim, Vicedecano de Investigaciones, al Dr. Henry Acuña, Jefe de la división de Investigación Científica, y a la Dra. Esperanza Fajardo, Directora del Centro de Investigaciones de la Facultad de Medicina. Este artículo es resultado del proyecto MED 735, financiado por el Fondo de investigaciones de la Universidad Militar Nueva Granada, por lo que agradecemos su apoyo.

Agradecemos a la Fundación Cardio Infantil, en especial al doctor Rodolfo Dennis, director del Departamento de Investigaciones por apoyar nuestro trabajo.

Agradecemos al Centro de Investigaciones de la Clínica del Country, en especial al Dr. Andrés Rodríguez, director del Centro de Investigaciones, al Dr. Jorge Ospina, Director Médico de la Clínica del Country, y al Dr. Tito Tulio Roa, Director de Educación Médica, por su apoyo a nuestras investigaciones.

\section{Referencias}

1. Peitgen H, Jurgens H, Saupe D. Chaos and fractals; new frontiers of science. New York: Springer-Verlag; 1992.

2. Peitgen $\mathrm{H}$. Strange attractors, the locus of chaos. En: Chaos and Fractals; new frontiers of science. New York: SpringerVerlag; 1992. p. 655-768.

3. Huikuri HV, Makikallio T, Peng CK, Goldberger A, Hintze U, Møller M. Fractal correlation properties of R - R interval dynamics and mortality in patients with depressed left ventricular function after and acute myocardial infarction. Circulation. 2000; 101:47-53.

4. Goldberger A, Amaral L, Hausdorff JM, Ivanov P, Peng Ch, Stanley HE. "Fractal dynamics in physiology: alterations with disease and aging". PNAS. 2002; 99:2466-2472.

5. Rodríguez J, Prieto S, Ortiz L, Bautista A, Bernal P, Avilán N. Diagnóstico Matemático de la Monitoria Fetal aplicando la ley de Zipf Mandelbrot. Rev Fac Med Univ Nac Colomb. 2006; 54(2):96-107.

6. Rodriguez J. Dynamical systems theory and ZIPF - Mandelbrot Law applied to the development of a fetal monitoring diagnostic methodology. XVIII Figo World Congress Of Gynecology And Obstetric. Kuala Lumpur, Malaysia. November 2006.

7. Rodríguez J. Entropía Proporcional De Los Sistemas Dinámicos Cardiacos: Predicciones físicas y matemáticas de la dinámica cardiaca de aplicación clínica. Rev Colomb Cardiol. 2010; 17:115-129. 
8. Rodríguez J, Prieto S, Bernal P, Isaza D, Salazar G, Correa $\mathrm{C}$, et al. Entropía proporcional aplicada a la evolución de la dinámica cardiaca. Predicciones de aplicación clínica. En: Caos y Complejidad en América Latina. En Prensa.

9. Rodríguez J, Correa C, Ortiz L, Prieto S, Bernal P, Ayala J. Evaluación matemática de la dinámica cardiaca con la teoría de la probabilidad. Rev Mex Cardiol. 2009; 20 (4):183-9.

10. Laplace P. Ensayo filosófico sobre las probabilidades. Barcelona: Altaya; 1995.

11. Feynman R, Leighton R, Sands M. Probabilidad. En: Feynman R, Leighton R, Sands M. Física. Vol. 1. Wilmington: AddisonWesley Iberoamericana, S. A. 1964. p. 6-1, 6-16.

12. Mood A, Graybill F, Boes D. Introduction to the theory of statistics. 3a Ed. Singapore: Mc. Graw-Hill; 1974.

13. Blanco L. Probabilidad, notas de clase. Universidad Nacional de Colombia. Departamento de Matemáticas y Estadística: 1996

14. Tsuji H, Larson M, Venditti F, et al. Impact of reduced heart rate variability on risk for cardiac events. The Framinghan Heart Study. Circulation. 1996; 94:2850-2855.

15. Nolan J, Batin P, Andrews R, et al. Prospective study of heart rate variability and mortality in chronic heart failure: results of the United Kingdom Heart Failure Evaluation and Assessment of Risk Trial (UK - heart). Circulation. 1998; 98:1510-1516.

16. Wolf M, Varigos G, Hunt D, Sluman J. Sinus arrhythmia in acute myocardial infarction. Med J Aus. 1978; 2:52-53.

17. Juha S. Perkio MaKi, Timo H. Mäkikallio, Heikki Huikuri. Fractal and complexity measures of heart rate variability. Clinical and Experimental Hypertension 2005; (2-3):149-158

18. Pontet J, Contreras P, Curbelo A, Medina J, Noveri S, Bentancourt S, et al. Heart Rate Variability as early marker of Multiple
Organ Dysfunction Syndrome in septic patients. Journal of Critical Care. 2003; 18(3): 156-163

19. Rodríguez J, Prieto S, Avilán N, Correa C, Bernal P, Ortiz L, et al. Nueva metodología física y matemática de evaluación del Holter. Rev Colomb Cardiol. 2008; 15:50-54.

20. Rodríguez J, Prieto S, Bernal P, Soracipa Y, Salazar G, Izasa D, Cruz LE, Correa C. Nueva metodología diagnóstica de la dinámica geométrica cardiaca. Dinámica cardiaca caótica del Holter. Rev Acad Colomb Cienc 2011; 35(134): 5-12.

21. Rodríguez J, Prieto S, Correa C, Bernal P, Puerta G, Vitery $\mathrm{S}$, et al. Theoretical generalization of normal and sick coronary arteries with fractal dimensions and the arterial intrinsic mathematical harmony. BMC Medical Physics. 2010; 10:1 http://www.biomedcentral.com/1756-6649/10/1.

22. Rodríguez J, Bernal P, Prieto S, Correa C. Teoría de péptidos de alta unión de malaria al glóbulo rojo. Predicciones teóricas de nuevos péptidos de unión y mutaciones teóricas predictivas de aminoácidos críticos. Inmunología 2010; 29(1):7-19.

23. Rodríguez J. Teoría de unión al HLA clase II teorías de Probabilidad Combinatoria y Entropía aplicadas a secuencias peptídicas. Inmunología. 2008; 27(4):151-166.

24. Rodríguez J. Método para la predicción de la dinámica temporal de la malaria en los municipios de Colombia. Rev Panam Salud Pública. 2010; 27(3):211-218.

25. Rodríguez J, Prieto S, Correa C, Posso H, Bernal P, Puerta G et al. Generalización Fractal de Células Preneoplásicas y Cancerígenas del Epitelio Escamoso Cervical. Una Nueva Metodología de Aplicación Clínica. Rev Fac Med. 2010; 18(2)173-181. 